\title{
Ballistic one-dimensional transport in InAs nanowires monolithically integrated on silicon
}

\author{
J. Gooth, ${ }^{1, a), b)}$ V. Schaller, ${ }^{1, a)}$ S. Wirths, ${ }^{1}$ H. Schmid, ${ }^{1}$ M. Borg, ${ }^{1}$ N. Bologna, ${ }^{1,2}$ S. Karg, ${ }^{1}$ \\ and H. Riel ${ }^{1, b)}$ \\ ${ }^{1}$ IBM Research - Zurich, Säumerstrasse 4, 8803 Rüschlikon, Switzerland \\ ${ }^{2}$ Electron Microscopy Center, EMPA, Swiss Federal Laboratories for Materials Science and Technology, \\ Überlandstrasse 129, 8600 Dübendorf, Switzerland
}

(Received 10 January 2017; accepted 8 February 2017; published online 21 February 2017)

\begin{abstract}
We present the monolithic integration and electrical characterization of InAs nanowires (NWs) with the well-defined geometries and positions on $\mathrm{Si}$ as a platform for quantum transport studies. Hereby, one-dimensional (1D) ballistic transport with step-like 1D conductance quantization in units of $2 e^{2} / h$ is demonstrated for NWs with the widths between $28 \mathrm{~nm}$ and $58 \mathrm{~nm}$ and a height of $40 \mathrm{~nm}$. The electric field control of up to four individual modes is achieved. Furthermore, the sub-band structure of the nanowires is investigated using bias spectroscopy. The splitting between the first and the second sub-band increases as the width of the NWs is reduced, whereas the degeneracy of the second sub-band can be tuned by the symmetry of the NW cross section, in accordance with a "particle in a box" model. The length-dependent studies reveal ballistic transport for up to $300 \mathrm{~nm}$ and quasi-ballistic transport with a mean free path of $470 \mathrm{~nm}$ for longer InAs NW channels at $30 \mathrm{~K}$. We anticipate that the ballistic 1D transport in monolithically integrated InAs NWs presented here will form the basis for sophisticated quantum wire devices for the future integrated circuits with additional functionalities. Published by AIP Publishing. [http://dx.doi.org/10.1063/1.4977031]
\end{abstract}

The III-V semiconductor nanowires (NWs) are very attractive prospects for the continued downscaling of complementary metal-oxide-semiconductor (CMOS) circuits because of their high bulk carrier mobilities and saturation velocities. Furthermore, they promise to provide value-added functionalities on integrated circuits, such as spintronic components, ${ }^{1-4}$ nonlinear circuit elements ${ }^{5,6}$ and topological quantum devices, ${ }^{7-12}$ which are increasingly important for meeting the demand for ever more powerful computational systems. Key premises for this are that ballistic electron transport can be achieved and that basic one-dimensional (1D) quantum confinement theory translates into the NW channels. In a ballistic 1D system, the motion of confined electrons is restricted to discrete energy bands according to Landauer's formalism, and the spacing of these energy bands is determined by the size and symmetry of the channel cross section. As routinely observed in the gate-defined quantum point contacts on two-dimensional systems, ${ }^{13,14}$ the hallmark of ballistic 1D transport in experiments is a step-wise increase in the electrical conductance $G$ in units of $2 e^{2} / h$, where each step corresponds to the (de-)population of an individual 1D sub-band ( $e$ is the elementary charge and $h$ is Planck's constant). The pre-factor 2 accounts for spin degeneracy. The signatures of 1D ballistic transport have also been observed in individual InAs, ${ }^{15-20} \mathrm{InSb}^{21,22} \mathrm{GaAs}^{23,24}$ and $\mathrm{In}_{x} \mathrm{Ga}_{x-1} \mathrm{As}^{25} \mathrm{NW}$ devices. However, despite the significant recent progress in the III-V NW epitaxial growth techniques, a systematic experimental study of how the geometrical parameters affect the electronic band structure and the positioning of the NW in a working environment remain challenging, ${ }^{26}$ because the precise control of the geometry,

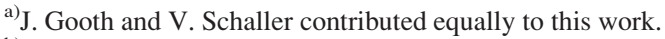

b)Electronic addresses: jog@zurich.ibm.com and hei@zurich.ibm.com
}

materials, and interfaces on the nanoscale is very difficult to achieve. Furthermore, low-cost and large-scale integration of III-V semiconductor NW quantum devices on the standard Si CMOS substrates, i.e., $\mathrm{Si}$ (100), is key for NWs to become a viable and competitive alternative to the state-of-the art Si-based technologies. In this regard, the main challenges to resolve are the defects due to the large lattice- and thermal expansion mismatch and the anti-phase boundaries originating from the semi-ionic nature of III-V crystals.

We have used the recently developed template-assisted selective epitaxy (TASE) ${ }^{27-31}$ method and present a systematic study on 1D quantum transport in a series of InAs NWs with tailored cross-sectional size and symmetry. TASE provides high-quality III-V nanostructures for monolithic integration on Si with low defect densities and surface roughness and allows very good control of the position, orientation and dimensions of NWs. The InAs NWs investigated are epitaxially grown, single-crystalline and free of threading dislocations. Contacted by two metal electrodes, the NW forms a 1D electron-conducting channel whose Fermi level can be controlled using the Si substrate as gate electrode. At room temperature, the NWs grown exhibit a field-effect mobility of up to $1700 \mathrm{~cm}^{2} \mathrm{~V}^{-1} \mathrm{~s}^{-1}$, illustrating the high quality of the material (see supplementary material for details). We start by characterizing quantum transport in the NW devices using the field-effect measurements at constant voltage bias and temperatures as low as $30 \mathrm{~K}$. In the second set of experiments, we investigate the 1D sub-band structure of the NWs via bias spectroscopy and test Landauer's formalism within a "particle in a box" picture. Finally, the temperature and ballistic length limits of the 1D NW devices are explored.

The NWs have been grown using the TASE process. ${ }^{27}$ The pre-defined $\mathrm{SiO}_{2}$ nanotube templates are used to control 
(a)

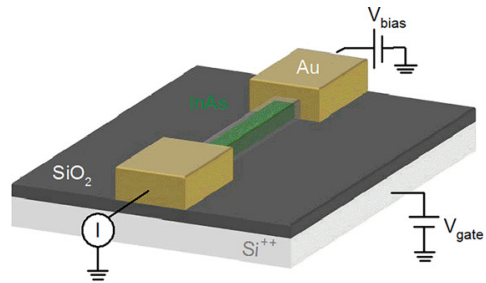

(b)

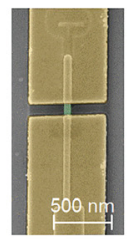

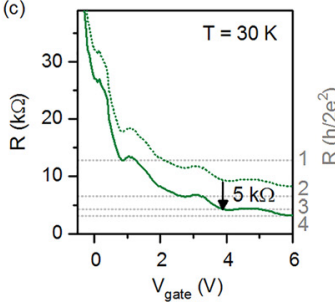

(d)

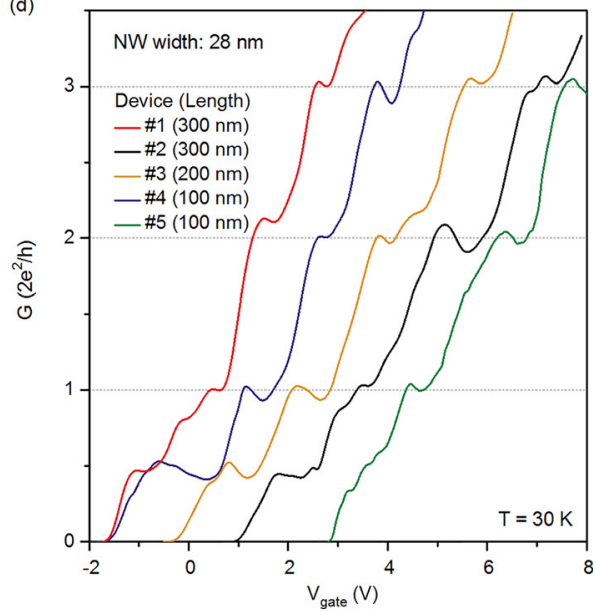

FIG. 1. Ballistic one-dimensional transport in InAs nanowires (NWs). (a) Schematic and (b) false-colored SEM image of a typical NW device. InAs NWs (green) are integrated on Si using template-assisted selective epitaxy (TASE). All NWs have a height of $40 \mathrm{~nm}$. Two-terminal Ni/Au (30 nm/50 nm) contacts allow the current $I$ to be measured in response to an applied bias voltage $V_{\text {bias. }}$ A 140 -nm-thick $\mathrm{SiO}_{2}$ layer serves as dielectric to apply a global back-gate voltage $V_{\text {gate }}$ (c) Subtraction of the contact resistance. Resistance $R=1 / G$ as a function of the gate voltage $V_{\text {gate }}$ (dotted line: raw measurement data; solid line: with the contact resistance subtracted). The numbers on the right axis are in units of $1 /\left(2 e^{2} / h\right)$. (d) Electrical conductance $G=I / V_{\text {bias }}$ as a function of $V_{\text {gate }}$ for five exemplarily 28-nm-wide NWs, measured at $30 \mathrm{~K}$ with a constant $V_{\text {bias }}=5 \mathrm{mV}$. The individual traces are corrected for the contact resistance and, for clarity reasons, shifted by \#• $1.5 \mathrm{~V}$ on the $x$-axis. All NWs with lengths below $300 \mathrm{~nm}$ (device \#1: $300 \mathrm{~nm}$, \#2: $300 \mathrm{~nm}$, \#3: $200 \mathrm{~nm}$, \#4: $100 \mathrm{~nm}$, \#5: $100 \mathrm{~nm}$ ) exhibit quantized 1D conductance plateaus.

the length, cross section, position and axial direction of the NW devices. The templates are filled with InAs from trimethylindium and tertiarybutylarsine precursors via metal organic vapor deposition at $550{ }^{\circ} \mathrm{C}$. The process parameters are adjusted such that the NW axis corresponds to the (110) axis of the InAs crystal. For electrical transport experiments, two metal electrodes are attached at the ends of each NW by means of standard e-beam lithography, sulfur passivation, evaporation of $\mathrm{Ni} / \mathrm{Au}(30 \mathrm{~nm} / 50 \mathrm{~nm})$ and a lift-off process. The template oxide is removed selectively only in the contact area. This is a specific advantage of TASE. Thanks to the remaining template oxide on the transport channel - the NW surface is protected in-situ and not exposed to air or any other chemical during the preparation processes. The two-terminal configuration is used to measure the current $I$ through the devices in response to an applied DC bias voltage $V_{\text {bias. }}$ A schematic measurement layout and a typical measurement platform are shown in Figs. 1(a) and 1(b), respectively. To control the Fermi energy $E_{\mathrm{F}}$, all samples are equipped with a global back-gate. We have measured 34 InAs NW devices with the widths of $a=28,38,48$ and $58 \mathrm{~nm}$ and a height of $b=40 \mathrm{~nm}$. Tuning the width $a$ (with the height $b$ being constant) enables the simultaneous investigation of the size and symmetry effects. The linear currentvoltage curves reveal ohmic contacts (see SM Fig. 2). The length of the NW channels is defined by the source-drain contact distance and is varied from $l=100 \mathrm{~nm}$ to $1200 \mathrm{~nm}$ to cover both ballistic and quasi-ballistic regimes.

In the first set of experiments, we measure the conductance $G$ of the NWs at $30 \mathrm{~K}$ and sweep the gate voltage $V_{\text {gate }}$ with a rate of $15 \mathrm{mV} / \mathrm{s}$ at a fixed DC voltage bias of $V_{\text {bias }}=5 \mathrm{mV}$ applied. The electrical conductance $G=I / V_{\text {bias }}$ of the device is then calculated directly from the measurement current $I$. To obtain only the NW channel resistance, a series resistance $R_{\text {series }}$ has been subtracted from all $G\left(V_{\text {gate }}\right)$ measurement data shown (Fig. 1(c)) to account for the contact and the lead resistance of the device. Following the procedure of van Weperen et al., ${ }^{22} R_{\text {series }}$ is determined for each device individually at $30 \mathrm{~K}$ in such a way that the conductance of the $n$th plateau is $n \cdot 2 e^{2} / h$. This method provides fairly accurate results as there are 3-4 conductance plateaus per $G-V_{\text {gate }}$ curve that have to be fitted with only one parameter. Series resistances of around $5 \pm 2 \mathrm{k} \Omega$ are obtained in accordance with the transmission-line experiments. ${ }^{32}$ We have measured $30 \mathrm{NW}$ devices with channel lengths from $100 \mathrm{~nm}$ to $300 \mathrm{~nm}$, randomly distributed over the $2 \mathrm{~cm} \times 2 \mathrm{~cm}$ chip. Of the $30 \mathrm{NW}$ devices with $l \leq 300 \mathrm{~nm}$ investigated, 28 show well-defined conductance plateaus at integer multiples at $G_{0}$, demonstrating ballistic $1 \mathrm{D}$ quantum transport ( $>90 \%$ yield). Fig. 1(d) exemplarily shows the recorded traces of the 28-nm-wide structures. For better comparison, the curves are shifted on the $x$-axis. To gain more insight into the ballistic 1D transport mechanism, we
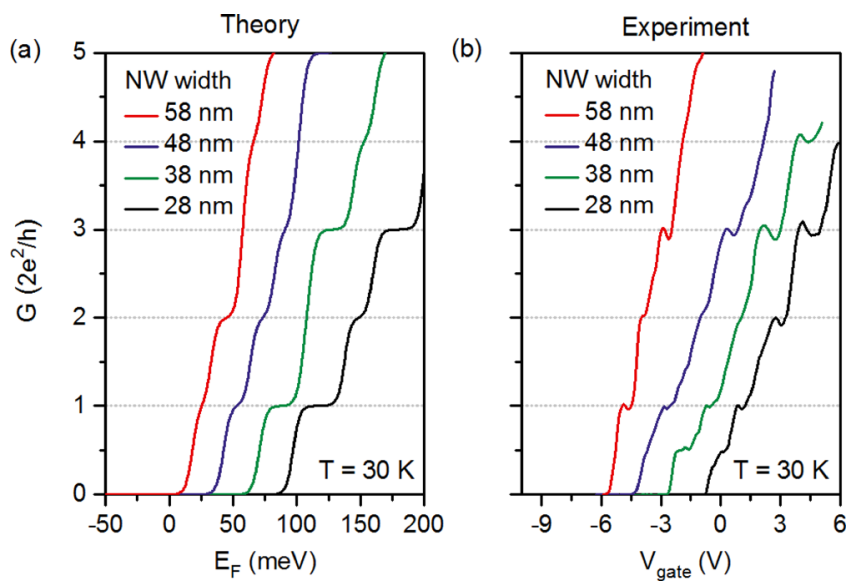

FIG. 2. Comparison of experimental data with the infinite potential-well model. (a) Calculated electrical conductance for the four NW diameters experimentally investigated at $30 \mathrm{~K}$ using Landauer's theory. (b) Corresponding measurement data. 
have also compared the experimental features observed with theory.

Within the Landauer formalism, ${ }^{17}$ the electrical conductance $G$ at temperature $T$ for infinitely small bias voltages is given by

$$
G(T)=\frac{2 e^{2}}{h} \sum_{n, m} \int_{\epsilon_{n, m}}^{\infty} \frac{d f\left(E, E_{\mathrm{F}}, T\right)}{d E} T(E) d E .
$$

Approaching a simple infinite square-well model, the eigenenergies of the system are given by

$$
\epsilon_{n, m}=\frac{\hbar^{2} \pi^{2}}{2 m^{*}}\left[\frac{n^{2}}{a^{2}}+\frac{m^{2}}{b^{2}}\right],
$$

where $n$ and $m$ are integer quantum numbers $(n, m=1,2,3$, $4, \ldots), \hbar=h / 2 \pi$ denotes the reduced Planck's constant, $f\left(E, E_{\mathrm{F}}, T\right)$ is the Fermi distribution and $m^{*}=0.023 m_{0}$ is the effective electron mass of InAs. Fig. 2(a) shows $G\left(E_{\mathrm{F}}\right)$ at $30 \mathrm{~K}$, calculated using Eqs. (1) and (2) in the case of perfect mode transmission $(T(E)=1)$ for all NW cross sections investigated. Because of the finite temperature and the increasing sub-band spacing with decreasing width, the conductance plateaus in general appear more pronounced, as the width of the potential well is reduced. For the most symmetric $(38 \mathrm{~nm} \times 40 \mathrm{~nm})$ well, the second plateau is not visible, whereas it is clearly visible for wider and thinner wells. As observed in the cylindrical NWs, ${ }^{16-18,21}$ rotational symmetry can give rise to additional degeneracies in the second subband $\left(\epsilon_{1,2}=\epsilon_{2,1}\right)$. At finite temperatures, sub-bands that are close in energy or fully degenerated by symmetry will be populated at similar values of $E_{\mathrm{F}}$, leading to a double step of $2 G_{0}$.

Fig. 2(b) shows the results of the corresponding transport experiments for four representative NWs with the widths of $28,38,48$, and $58 \mathrm{~nm}$ and a channel length of $300 \mathrm{~nm}$. As predicted by theory, the $1 \mathrm{D}$ quantum features get weaker as the NW width increases and the second plateau of the nearsymmetric cross-sectional wire almost disappears. Other features, such as the third plateau, seem even more pronounced than expected from the calculations, and the plateaus exhibit an additional substructure. Differences between the numerical simulations and the experimental data indicate a modification of the sub-bands under electrostatic gating ${ }^{21}$ as well as highorder terms in the Fermi velocity. ${ }^{19}$ Moreover, we observe an additional $0.5 G_{0}$ conductance plateau for the 28-nm, 38-nm and 48-nm-wide NWs, which is not covered by Eq. (1). Such half-integer features indicate a spin splitting of the first plateau, ${ }^{23,24,33-35}$ which may originate from a strong spin-orbit coupling combined with an asymmetric confinement potential, electron-electron interaction, or spin-selective injection from the ferromagnetic Ni contacts.

For a more quantitative analysis of the sub-band structure, we have performed bias spectroscopy ${ }^{15,21}$ on NWs of all four widths. The differential conductance $G=\mathrm{d} I / \mathrm{d} V_{\text {bias }}$ is measured as a function of $V_{\text {gate }}$ and $V_{\text {bias }}$, and the series resistance is subtracted. The data for the 28-nm- and the 58-nm-wide NWs are shown exemplarily in Figs. 3(a) and 3(b), respectively. In all devices, we observe a second feature of $1 \mathrm{D}$ quantum transport. ${ }^{15}$ As most clearly apparent in the derivative of the conductance with respect to the gate voltage $g=\mathrm{d} G / \mathrm{d} V_{\text {bias }}$ (see Figs. 3(c) and 3(d)), the diamondlike structures in the nonlinear conductance are formed by the plateaus of multiple integers of $G_{0}$ around zero $V_{\text {bias }}$. Furthermore, the so-called half-plateaus of intermediate values of the zero-bias conductance steps appear at high $V_{\text {bias }}$. Such half-plateaus are caused by the occupation of different sub-bands. The conductance will only be quantized in the integer multiples of $G_{0}$ if the same single $1 \mathrm{D}$ state lies between the chemical potentials of source and drain. Consequently, when correcting for the voltage drop across the series resistance, the tip of the corresponding diamonds ( $V_{\text {bias,tip }}$ ) will reflect the spacing between sub-bands $i$ and $j$ :

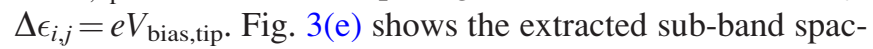
ing and compares it with the theoretical sub-band spacing of an infinite square well (Eqs. (1) and (2)). Both size and symmetry effects are clearly visible in the experimental data, as expected from the simple "particle in a box" model.
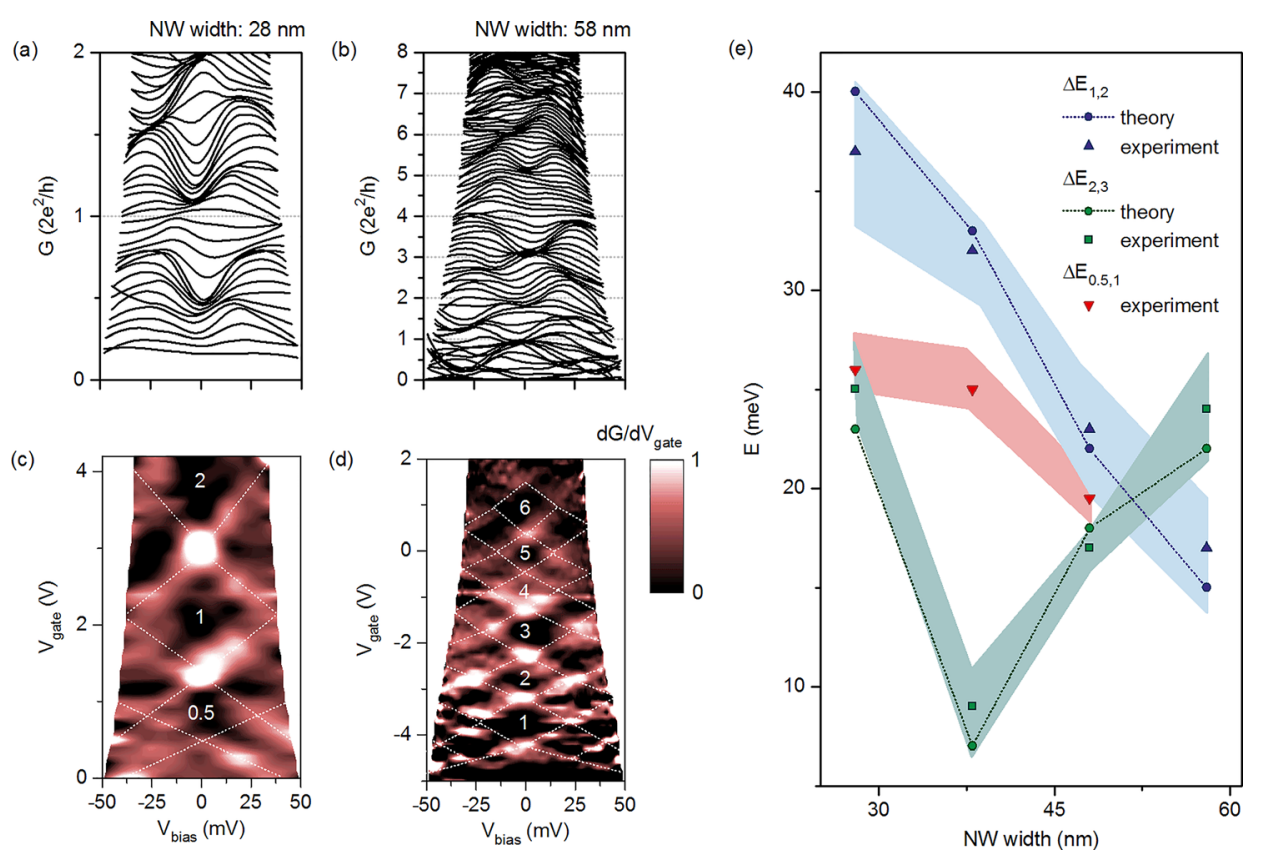

FIG. 3. Bias spectroscopy of the 1D sub-band structure at $30 \mathrm{~K}$. (a) Biascorrected differential conductance $G$ $=\mathrm{d} I / \mathrm{d} V_{\text {bias }}$, exemplarily shown for a $28-$ $\mathrm{nm}$ and (b) a 58-nm-wide NW. Plateaus of multiple integer of $G_{0}$ around zero $V_{\text {bias }}$ and half-plateaus of intermediate values at high $V_{\text {bias }}$ are observed (data of a 38-nm and a 48-nm-wide NW are given in the SM). (c) and (d) The corresponding derivative of the conductance with respect to the gate voltage $g=\mathrm{d} G$ / $\mathrm{d} V_{\text {gate }}$ reveals diamond-like structures whose width directly reflects the distance between the individual sub-bands. (e) Comparison of the experimentally extracted sub-band spacing and the infinite potential-well model. The shaded area reflects the error of the experimental data, estimated from the average of the negative and the positive bias. 
Whereas $\Delta \epsilon_{1,2}$ is not affected by the symmetry effects and increases as the NW width decreases owing to enhanced confinement, $\Delta \epsilon_{2,3}$, in contrast, is sensitive to the degeneration of the second and the third sub-band and approaches a minimum at the NW cross section of highest symmetry $(38 \mathrm{~nm} \times 40 \mathrm{~nm})$. The spin-splitting $\Delta \epsilon_{0.5,1}$ increases with decreasing NW width. These results demonstrate very good control over the quantum confinement conditions in InAs NWs fabricated using TASE. Exploiting the ability to systematically tune the NW cross section allowed us to show that the quantum confinement is well described by basic transport theory.

Finally, we explore the length and temperature limits of the ballistic 1D transport in our 28-nm-wide devices. To determine the ballistic length of 28 -nm-wide NWs at $30 \mathrm{~K}$, $G\left(V_{\text {gate }}\right)$ is measured for devices with channel lengths between $100 \mathrm{~nm}$ and $1200 \mathrm{~nm}$, all on the same chip (Fig. 4(a)). $V_{\text {gate }}$ is swept between 0 and $6 \mathrm{~V}$, and a fixed voltage bias of $V_{\text {bias }}=5 \mathrm{mV}$ is applied. On all traces, a contact resistance of $5 \mathrm{k} \Omega$ has been subtracted. For a qualitative analysis, the center of the conductance plateaus is identified by the minima of the corresponding derivative of the conductance with respect to $V_{\text {gate }}$ (Fig. 4(b)). In this way, the height of the individual sub-band plateaus can be compared (see Fig. 4(c)). We find

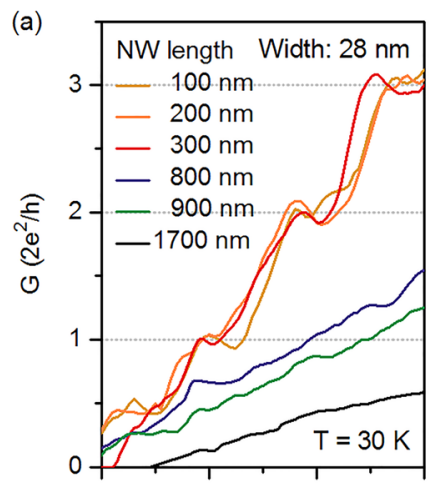

(c)

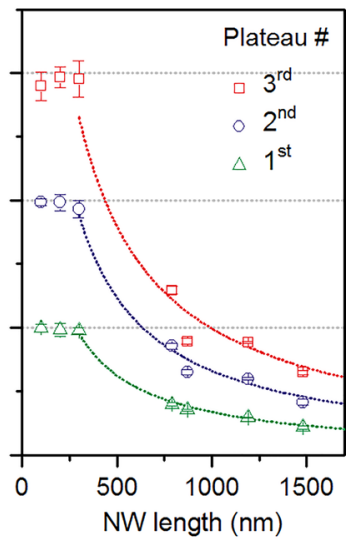

(b)
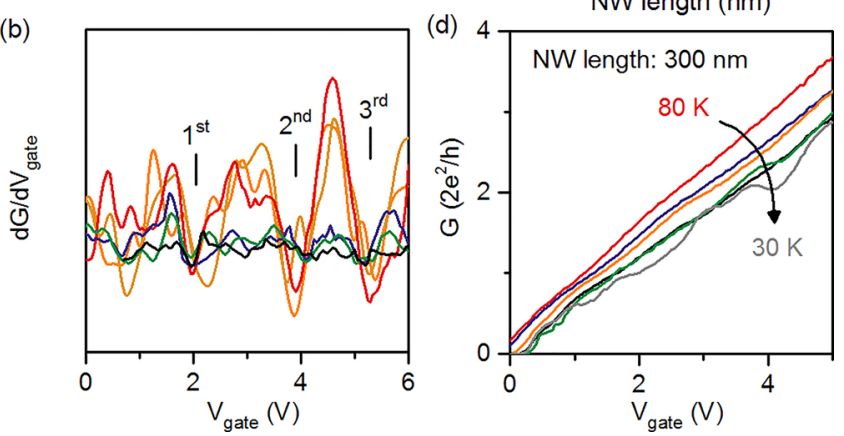

FIG. 4. Length and temperature limits of the ballistic 1D transport. (a) Electrical conductance $G$ as a function of $V_{\text {gate }}$ for individual 28-nm-wide NW devices with various lengths. (b) Corresponding derivative of the conductance with respect to $V_{\text {gate }}$ (c) Experimental plateau conductance (symbols) as a function of channel length $l$. The values are obtained by averaging the data in (a), over the full width at half maximum of the minima marked in (b). The error of the data in (c) reflects the standard deviation across the plateau. Up to 300-nm channel length, the NWs remain ballistic. Mean free paths of $\lambda=(473 \pm 81)$ for the first, $\lambda=(242 \pm 41) \mathrm{nm}$ for the second and $\lambda=(156$ $\pm 27) \mathrm{nm}$ for the third sub-band are obtained from fits in the quasi-ballistic regime (lines) of $G=G_{0}^{-1}(1+l / \lambda)^{-1}$. (d) Temperature dependence. $G\left(V_{\text {gate }}\right)$ of a $300-\mathrm{nm}$-long device at temperatures from $30 \mathrm{~K}$ to $80 \mathrm{~K}$, in $10 \mathrm{~K}$ steps. that up to a channel length of $300 \mathrm{~nm}$ the height of the plateaus remains at integer multiples of $G_{0}$, indicating the ballistic transport. However, for the $800-\mathrm{nm}, 900-\mathrm{nm}$ and 1200-nm-long devices, we observe signatures for the onset of scattering, as the conductance decreases monotonically with increasing length $l$. Within the Landauer picture, ${ }^{17}$ the conductance in this quasi-ballistic regime can be expressed as $G=G_{0}{ }^{-1}(1+l / \lambda)^{-1}$, where $\lambda$ is the mean free path of the system. Fitting our measurement data, we obtain a mean free path of $\lambda=(473 \pm 81) \mathrm{nm}$ for the first, $\lambda=(242 \pm 41) \mathrm{nm}$ for the second and $\lambda=(156 \pm 27) \mathrm{nm}$ for the third sub-band. The decreasing mean free path with increasing sub-band index indicates energy-dependent scattering, in agreement with a modification of the sub-bands under electrostatic gating. ${ }^{21}$

To investigate the temperature limit in our devices further, $G\left(V_{\text {gate }}\right)$ measurements at a constant $V_{\text {bias }}=5 \mathrm{mV}$ have been carried out between $30 \mathrm{~K}$ and $200 \mathrm{~K}$ on NWs with a channel length of $300 \mathrm{~nm}$. The data are shown in Fig. 4(d). For the 28-nm-wide NWs, the quantum features remain visible up to $70 \mathrm{~K}$ but degrade at elevated temperatures. The temperature broadening of the Fermi distribution is about $\Delta E_{\mathrm{F}}=4 \mathrm{k}_{\mathrm{B}} T$, posing a theoretical temperature limit for distinguishing the individual sub-bands. At $80 \mathrm{~K}$, the broadening factor is $\Delta E_{\mathrm{F}}=28 \mathrm{meV}$, which compares well with the sub-band spacing of the NW devices investigated, as shown in Fig. 3(e). Note that in diffusive systems, inelastic scattering by, e.g., phonons, is expected to increase with increasing temperature, leading to an additional widening of the energy states and thus to a decreased temperature window for observing the $1 \mathrm{D}$ features. We therefore conclude that our 28-nm-wide and $300 \mathrm{~nm}$-long NW devices remain ballistic at least up to $70 \mathrm{~K}$. Smaller and thinner wires may allow roomtemperature ballistic quantum transport and are in principle achievable with TASE.

In conclusion, we have demonstrated the monolithic integration of ballistic 1D InAs NW quantum devices on $\mathrm{Si}$. The TASE process applied is CMOS-compatible, with an excellent control of the NW position and size confinement. A series of NWs with $40 \mathrm{~nm}$ height, widths between $28 \mathrm{~nm}$ and $58 \mathrm{~nm}$ and channel lengths from $100 \mathrm{~nm}$ to $1200 \mathrm{~nm}$ have been fabricated, enabling the systematic study of quantization in NWs. Conductance quantization in the steps of integer multiples of $2 e^{2} / h$ has been observed up to $70 \mathrm{~K}$ in more than $90 \%$ of the devices with a channel length below $300 \mathrm{~nm}$, indicating the ballistic transport. The quasi-ballistic transport is obtained for channel lengths of up to $1200 \mathrm{~nm}$, revealing a mean free path of $473 \mathrm{~nm}$. The quantum confinement in rectangular InAs NWs seems to be well reflected by a simple "particle in a box" model. The future experiments involving magneto-transport studies to investigate the $g$-factor of these 1D materials are anticipated. In depth understanding of the device electrostatics ${ }^{36}$ including the quantum capacitance ${ }^{37}$ is very desirable to predict its performance limits. Also, the exact nature of the $0.5 G_{0}$ feature, attributed to a spin nondegeneracy, should be further investigated. We believe that TASE provides a versatile platform for developing sophisticated quantum wire devices for use in the fields of quantum computing, ${ }^{7-12}$ spintronics ${ }^{1-4}$ and nonlinear electronics. ${ }^{5,6}$ 
See supplementary material for the room-temperature field-effect analysis, current-voltage characteristics, additional bias-spectroscopies and a transmission electron micrograph of a nanowire cross section.

The authors acknowledge W. Riess, K. Moselund, B. Mayer, and B. Gotsman for the fruitful discussions and J. Finley for the support. We also thank C. Bollinger for copyediting and M. Tschudy for metallization. The research leading to these results has received funding from the European Union's Horizon 2020 research and innovation program under grant agreement No 687931 "REMINDER," the European Union Seventh Framework Programs (FP7/ 2007-2013), No. 619509 "E2SWITCH," the Marie Curie Post-Doctoral Research Fellowship No. 704045 "MODES," and also the Rudolf Diesel Industry Fellowship funded by the EU Marie Curie Cofund Program (FP7-MC-COFUND, Grant Agreement No. 291763).

${ }^{1}$ D. Liang and X. P. A. Gao, Nano Lett. 12, 3263 (2012).

${ }^{2}$ F. Rossella, A. Bertoni, D. Ercolani, M. Rontani, L. Sorba, F. Beltram, and S. Roddaro, Nat. Nanotechnol. 9, 997 (2014).

${ }^{3}$ S. S. Hong, Y. Zhang, J. J. Cha, X. L. Qi, and Y. Cui, Nano Lett. 14, 2815 (2014).

${ }^{4}$ S. Nadj-Perge, S. M. Frolov, E. P. Bakkers, and L. P. Kouwenhoven, Nature 468, 1084 (2010).

${ }^{5}$ M. Büttiker, Phys. Rev. Lett. 57, 1761 (1986).

${ }^{6}$ P. Stano and P. Jacquod, Phys. Rev. Lett. 106, 206602 (2011).

${ }^{7}$ M. T. Deng, C. L. Yu, G. Y. Huang, M. Larsson, P. Caroff, and H. Q. Xu, Nano Lett. 12, 6414 (2012).

${ }^{8}$ A. Das, Y. Ronen, Y. Most, Y. Oreg, M. Heiblum, and H. Shtrikman, Nat. Phys. 8, 887 (2012).

${ }^{9}$ S. M. Albrecht, A. P. Higginbotham, M. Madsen, F. Kuemmeth, T. S. Jespersen, J. Nyg, P. Krogstrup, and C. M. Marcus, Nature 531, 206 (2016).

${ }^{10}$ V. Mourik, K. Zuo, S. M. Frolov, S. R. Plissard, E. P. A. M. Bakkers, and L. P. Kouwenhoven, Science 336, 1003 (2012).

${ }^{11}$ L. P. Rokhinson, X. Liu, and J. K. Furdyna, Nat. Phys. 8, 795 (2012).

${ }^{12}$ J. Alicea, Y. Oreg, G. Refael, F. von Oppen, and M. P. A. Fisher, Nat. Phys. 7, 412 (2011).

${ }^{13}$ B. J. van Wees, L. P. Kouwenhoven, E. M. M. Willems, C. J. P. M. Harmans, J. E. Mooij, H. van Houten, C. W. J. Beenakker, J. G. Williamson, and C. T. Foxon, Phys. Rev. B 43, 12431 (1991).

${ }^{14}$ D. Tobben, D. A. Wharam, G. Abstreiter, J. P. Kolthaus, and F. Schaffler, Semicond. Sci. Technol. 10, 711 (1995).

${ }^{15}$ S. Heedt, W. Prost, J. Schubert, D. Grützmacher, and T. Schäpers, Nano Lett. 16, 3116 (2016).
${ }^{16}$ A. C. Ford, S. B. Kumar, R. Kapadia, J. Guo, and A. Javey, Nano Lett. 12, 1340 (2012).

${ }^{17}$ S. Chuang, Q. Gao, R. Kapadia, A. C. Ford, J. Guo, and A. Javey, Nano Lett. 13, 555 (2013).

${ }^{18}$ S. Abay, D. Persson, H. Nilsson, H. Q. Xu, M. Fogelström, V. Shumeiko, and P. Delsing, Nano Lett. 13, 3614 (2013).

${ }^{19}$ P. Mensch, S. Karg, V. Schmidt, B. Gotsmann, H. Schmid, and H. Riel, Appl. Phys. Lett. 106, 93101 (2015).

${ }^{20}$ S. Heedt, A. Manolescu, G. A. Nemnes, W. Prost, J. Schubert, D. Grützmacher, and T. Schäpers, Nano Lett. 16, 4569 (2016).

${ }^{21}$ J. Kammhuber, M. C. Cassidy, H. Zhang, Ö. Gül, F. Pei, M. W. A. de Moor, B. Nijholt, K. Watanabe, T. Taniguchi, D. Car, S. R. Plissard, E. P. A. M. Bakkers, and L. P. Kouwenhoven, Nano Lett. 6, 3482 (2016).

${ }^{22}$ I. Van Weperen, S. R. Plissard, E. P. A. M. Bakkers, S. M. Frolov, and L. P. Kouwenhoven, Nano Lett. 13, 387 (2013).

${ }^{23}$ R. Crook, J. Prance, K. J. Thomas, S. J. Chorley, I. Farrer, D. A. Ritchie, M. Pepper, and C. G. Smith, Science 312, 1359 (2006).

${ }^{24}$ C. P. Scheller, T. M. Liu, G. Barak, A. Yacoby, L. N. Pfeiffer, K. W. West, and D. M. Zumbühl, Phys. Rev. Lett. 112, 066801 (2014).

${ }^{25}$ C. B. Zota, D. Lindgren, L.-E. Wernersson, and E. Lind, ACS Nano 9, 9892 (2015).

${ }^{26}$ P. M. Wu, J. Gooth, X. Zianni, S. F. Svensson, J. G. Gluschke, K. A. Dick, C. Thelander, K. Nielsch, and H. Linke, Nano Lett. 13, 4080 (2013).

${ }^{27}$ H. Schmid, M. Borg, K. Moselund, L. Gignac, C. M. Breslin, J. Bruley, D. Cutaia, and H. Riel, Appl. Phys. Lett. 106, 233101 (2015).

${ }^{28}$ M. Borg, H. Schmid, K. E. Moselund, D. Cutaia, and H. Riel, J. Appl. Phys. 117, 144303 (2016).

${ }^{29}$ M. Borg, H. Schmid, K. E. Moselund, G. Signorello, L. Gignac, J. Bruley, C. Breslin, P. Das Kanungo, P. Werner, and H. Riel, Nano Lett. 14, 1914 (2014).

${ }^{30}$ L. Czornomaz, E. Uccelli, M. Sousa, V. Deshpande, V. Djara, D. Caimi, M. D. Rossell, R. Erni, and J. Fompeyrine, in 2015 Symposium on VLSI Technology (VLSI Technology, 2015), p. T172.

${ }^{31}$ H. Schmid, D. Cutaia, J. Gooth, S. Wirths, N. Bologna, K. E. Moselund, and H. Riel, in 2016 IEEE International Electron Devices Meeting (2016), p. 3.6.1.

${ }^{32}$ S. Karg, V. Schaller, A. Gaul, K. Moselund, H. Schmid, B. Gotsmann, J. Gooth, and H. Riel, in 2016 46th European Solid-State Device Research Conference (2016), p. 341.

${ }^{33}$ P. Debray, S. M. S. Rahman, J. Wan, R. S. Newrock, M. Cahay, A. T. Ngo, S. E. Ulloa, S. T. Herbert, M. Muhammad, and M. Johnson, Nat. Nanotechnol. 4, 759 (2009).

${ }^{34}$ O. M. Auslaender, H. Steinberg, A. Yacoby, Y. Tserkovnyak, B. I. Halperin, K. W. Baldwin, L. N. Pfeiffer, and K. W. West, Science (80-) 308, 88 (2005).

${ }^{35}$ M. Kohda, S. Nakamura, Y. Nishihara, K. Kobayashi, T. Ono, J. Ohe, Y. Tokura, T. Mineno, and J. Nitta, Nat. Commun. 3, 1082 (2012).

${ }^{36}$ A. Ajaykumar, X. Zhou, S. B. Chiah, and B. Syamal, IEEE Electron Device Lett. 37, 1051 (2016).

${ }^{37}$ M. Abul Khayer and R. K. Lake, IEEE Trans. Electron Devices 56, 2215 (2009). 